\title{
Quaderni
}

QUADERNI Communication, technologies, pouvoir

67| Automne 2008

Jeu vidéo et discours

\section{Matrix ou le sablier de nos représentations}

\section{David Morin Ulmann}

\section{(2) OpenEdition \\ Journals}

\section{Édition électronique}

URL : http://journals.openedition.org/quaderni/235

DOI : $10.4000 /$ quaderni.235

ISSN : 2105-2956

\section{Éditeur}

Les éditions de la Maison des sciences de l'Homme

\section{Édition imprimée}

Date de publication : 5 octobre 2008

Pagination : 101-106

Référence électronique

David Morin Ulmann, "Matrix ou le sablier de nos représentations », Quaderni [En ligne], 67 | Automne 2008, mis en ligne le 05 octobre 2008, consulté le 19 avril 2019. URL : http://journals.openedition.org/ quaderni/235 ; DOI : 10.4000/quaderni.235 


\section{Matrix \\ ou le \\ sablier \\ de nos \\ représentations}

David Morin

Ulmann

docteur en sociologie chargé de cours en sociologie Universités de Nantes et d'Angers

\section{Matrix ou la Pharmacie des Idiots}

Jacques Derrida, dès le premier paragraphe de $L a$ Pharmacie de Platon (1972), écrit : «Un texte n'est un texte que s'il cache au premier regard, au premier venu, la loi de sa composition et la règle de son jeu. (...) Un texte reste d'ailleurs toujours imperceptible. La loi et la règle ne s'abritent pas (...) dans l'inaccessible d'un secret, simplement elles ne se livrent jamais, au présent, (...) à rien $q u$ 'on puisse rigoureusement nommer perception. Au risque toujours et par essence de se perdre ainsi définitivement. » Je crois ce paragraphe utile lors d'une analyse du film américain Matrix (1999). D'abord, parce que tout objet a trois moments : production-fabrication, circulation-distribution, consommation. En sociologie des œuvres, on parle aussi de réception ou de sociologie des publics, c'est-à-dire sociologie des conditions de réception de certains produits culturels par certains publics. Par exemple, il y a une sociologie historique de la réception de la philosophie (Fabiani, 1988), une sociologie de la réception du groupe Metallica (Hein, 2003), une sociologie de la réception des films d'action (Gimello, 2007). On comprend l'objet en comprenant son " circuit », son économie politique (David Morin Ulmann, thèse de doctorat, Nantes, 2004). Ces sociologies s'intéressent aux conditions et aux «qualités empruntées », aux caractéristiques socio-économiques et psychosociologiques des publics qui reçoivent et consomment des produits culturels. Mais ces sociologies n'essentialisent jamais la consommation de ces produits, n'essentialisent jamais le goût, les qualités empruntées des consommateurs.

Nous devons évidemment comprendre que l'in- 
troduction de Derrida porte une contradiction, à moins qu'il ne s'agisse d'un mode d'emploi, d'une règle à suivre : son texte même nous sera incompréhensible au premier regard... Mais qu'est-ce qu'un « second regard » ? Est-ce celui, promu par Descartes, des yeux de l'esprit plutôt que le regard du corps ? On peut le penser. Et pour Matrix, ce sera la même chose : il faudra le regarder avec les yeux de la culture, des yeux tout à fait impersonnels, les yeux des idées. Derrida parle encore de « premier venu ». Qui est-il, celui-là ? Est-ce ce crétin dont parle Slavoj Zizek dans les toutes premières lignes d'un texte sur Matrix : «Lorsque je suis allé voir Matrix dans un cinéma en Slovénie, j'ai eu la chance unique de m'asseoir à côté du spectateur idéal pour ce film - à savoir, un idiot. » (2004) Derrida et Zizek partagent la même « délicatesse de goût » quant à la perception des choses. Philosophes, ils s'attachent à l'analyse interne de l'objet, c'est-à-dire aux rapports proprement culturels que l'objet analysé favorise en leur for intérieur. Manque toutefois l'analyse externe de l'objet, celle de la production et de consommation « par les gens » : analyse des conditions de possibilités socio-historiques, «psychologiques », dudit texte et dudit film. Car le piège du commentaire interne - dans lequel, pour la « démonstration », je peux bien tomber -, c'est qu'il ne se réfère qu'à l'objet analysé et à son double, son commentaire. Dès lors, dans tout commentaire interne, il y a quelque chose d'égyptien, au sens antique, au sens où chaque signe du monde reçoit une explication, une place ; quelque chose d'égyptien au sens d'une détermination inéluctable du signifiant ${ }^{1}$, d'une prédestination de la signification : on y glisse tout ce que l'on croit y voir, tout ce que l'on veut y voir. Tout semble déjà écrit, promis, comme dans les dialogues entre Neo et l'Oracle.

À l'instar de toute "théorie du complot», il y a négation de l'aléatoire ; in fine, de l'Histoire. Cette «qualité égyptienne» des objets d'art, plus exactement des commentaires relatifs à ces objets spécifiques au champ de la culture, on la devine précocement dans l'interrogation d'élèves à leur professeur de français : "Vraiment, m'sieur, Baudelaire, il avait tout vu c'que vous avez vu vous? " Face à cette propriété-miroir de l'analyse interne, l'histoire de l'art, la sociologie de l'art et la sociologie des publics sont de bonnes méthodes d'analyses externes croisées... Toutefois la question posée par Derrida et Matrix revient vite : le commentaire excède-t-il l'objet commenté ? C'est plus largement, je crois, la question des représentations sociales, du mouvement pratique du passage de la société à la personne, balancier du collectif à l'individuel et réciproquement. Autre formulation : qu'est-ce qu'une œuvre? Réponse brute : une œuvre est un objet qui fait parler; or les autres objets ne font pas parler les siècles... Les dadaïstes avaient compris cela : ainsi n'utilisaient-ils plus les objets, mais les exposaient-ils dans des galeries afin qu'on parlât d'eux, et des dadaïstes. Ainsi, s'il n'y a pas d'inaccessible secret des œuvres, comme l'écrit Derrida, il y a que les œuvres ne se livrent pas « au premier venu » et « au premier regard ». Elles sont comme les coquettes, tout en retenue, disent « et oui et non », chaloupent, se donnent un peu et se refusent, surtout ne se livrent pas dans leur présent, en public - là est certainement l'astuce derridienne -, dans le moment de leur première exposition. Mais alors, quand se livrent-elles ? À l'abri, à l'abri du regard du premier venu et du premier regard. Les œuvres 
se livrent en privé. Les œuvres ne se livrent donc $q u$ 'à la culture, $q u$ 'au système de la culture, $q u$ 'à la totalité de la culture. Bref, l'objet n'a rien de coquet contrairement à l'objet d'art; cela nous le savions déjà, puisque, dans les églises, les tableaux étaient voilés et que L'origine $d u$ monde de Gustave Courbet le fut aussi... Peut-on finalement percevoir un texte et un film? Oui, mais pas sur le moment; sur le moment on est le premier venu, un idiot. Ce n'est ni le temps de la compréhension ni du commentaire ; c'est celui de la réception «naïve », gratuite, signifiante dans l'instant même de la réception des signifiés ${ }^{2}$. C'est seulement lorsque je regarde l'objet d'art avec la totalité de la culture - c'est-à-dire avec recul - que j'y vois quelque chose ; et c'est seulement lorsque je me laisse regarder par lui que je perçois ce qu'il a à me dire.

\section{Marx au cour de la Matrice}

En une cabriole, je laisse ici les analyses du collectif de la Machine philosophique $(2002)^{3}$; je laisse les trente pages de S. Zizek sur Lacan et « Malebranche à Hollywood» (2004); je laisse les trente-cinq pages d'Olivier Pourriol et son héros Descartes (2008). Je laisse mais médite sur cet aréopage : comment ces intellectuels n'ont-ils pas vu la morale de Marx dans les « représentations collectivisées » de Matrix ?... D'abord, faisons un lacanisme, car c'est écrit en toutes lettres : ôtez le $\mathrm{T}$ et le I, reste Marx... Extrayons à présent un passage central, autour de la vingt-cinquième minute du film, lorsque, à l'intérieur même de la Matrice, Morpheus rencontre et converse avec Neo : «- Sais-tu exactement de quoi je parle? demande Morpheus - De la Matrice, répond Neo - Veux-tu également... savoir ce qu'elle est? (Neo fait un signe d'acquiescement.) La Matrice est universelle. Elle est omniprésente. Elle est avec nous ici en ce moment même. Tu la vois chaque fois que tu regardes par la fenêtre ou lorsque tu allumes la télévision. Tu ressens sa présence quand tu pars travailler, quand tu vas à l'église ou quand tu paies tes factures. Elle est le monde qu'on superpose à ton regard pour t'empêcher de voir la vérité. - Quelle vérité ? demande Neo. - Le fait que tu es un esclave, Neo. Comme les autres, tu es né enchaîné... ", répond Morpheus. À la quarantième minute, sur un fond blanc étincelant, dans un espace comme abstrait, lors du tout premier retour de Neo dans la Matrice, Morpheus, une télécommande à la main, l'interroge sur le Réel. Puis il assène : «Voici le monde tel qu'il existe aujourd'hui. Bienvenue dans le désert du réel. » Morpheus terminera ce moment d'initiation pour Neo, en ajoutant: "Qu'est-ce que la Matrice ?... Le contrôle absolu. La Matrice est la simulation d'un monde imaginaire... créé dans le seul but de nous maintenir sous contrôle... jusqu'à ce que nous soyons tous transformés... en ceci. »Et Morpheus de nous montrer une pile.

En un mot, Matrix parle de la vieille difficulté à comprendre le processus de va-et-vient ou «passage » à la fois pratique et mental des représentations collectives aux représentations individuelles, et réciproquement. Matrix parle donc du sablier de nos représentations, de l'incarnation des pensées, de l'« histoire biologique des idées » ${ }^{4}$. Ce film parle donc de câbles et de membranes, évidemment d'interfaces... L'impact cognitif et l'effet structurant des représentations collectives se laissent facilement saisir si l'on regarde les jeunes enfants regarder les dessins animés virtuels : «J'adore ces gags! », sourit 
la petite fille à la fin d'un Barbie 3D, gags d'un making of imaginaire reprenant des scènes faussement ratées dudit dessin animé. De fait, ce que nous apprennent les images virtuelles, c'est que les représentations collectives n'ont pas besoin du vrai visage, physique, plastique, de la douleur et de la joie. Comme dans le théâtre grec, les personnages des animations n'ont pas de pantomime, pas de faciès : ils jouent la vie et les sentiments avec un masque géométrique, et les enfants s'en soucient aussi peu que s'en souciaient les Grecs.

\section{La Matrice comme tableau de la contrainte sociale}

Autre formulation de ce processus de va-et-vient entre les représentations collectives et individuelles : qu'est-ce qui, dans la société, nous fait obéir? David Hume pose que le respect est une passion, qu'on n'obéit pas par raison, que la raison n'est pas motrice du respect; ce que voudra contrarier Kant. Ainsi, dans le Traité de la nature humaine, III, « La morale », il conclut : « en ellemême, la raison est inactive (...) parfaitement inerte et se montre impuissante à prévenir comme à produire une action ou une inclination. » (1993) Deux siècles plus tard, en 1939, Norbert Elias, dans son Esquisse d'une théorie de la civilisation, explique que « la stabilité particulière des mécanismes d'autocontrainte psychique qui constitue le trait typique de l'habitus de l'homme "civilisé » est étroitement liée à la monopolisation de la contrainte physique et à la solidité croissante des organes sociaux centraux. " Monopolisation : c'est-à-dire la police et l'armée. Organes sociaux centraux : c'est-à-dire la formation d'un État. Faisant écho tant à Kant et Durkheim qu'à
Freud, il écrit plus loin : "dans un certain sens, le champ de bataille a été transporté dans le for intérieur de l'homme. (...) les pulsions, les émotions passionnés qui ne se manifestent plus directement dans la lutte entre les hommes, se dressent souvent à l'intérieur de l'individu contre la partie « surveillée » de son Moi. » (1975). Précisons encore : comment, depuis leur extériorité, les faits sociaux affectent-ils la personne afin qu'elle participe (être poussée par), et anticipe (être préoccupée par), et partage (être avec) un ensemble social ? - Qu'a donc de spécialement prestigieux la contrainte sociale? Qu'est-ce qui motive et « transcende » la personne, l'entraîne, lui fait respecter les institutions ? Le problème de l'intériorisation de la contrainte, Durkheim le « résout » tant empiriquement (avec l'ethnologie et la sociologie religieuse) que philosophiquement en préférant les conclusions de Hume (on ne peut agir par sens du devoir que s'il y a une autorité supérieure à l'individu) à celles de Kant (la raison comme " dieu intérieur » nous y pousse).

\section{Matrix, une sorte de mai 68 théorique}

Voilà pour le sablier des représentations, sablier qui n'a ni haut ni bas, qui n'a pas de sens, si l'on veut, sablier qui, quoique renversé, revient toujours à lui-même : à la contrainte-système et, esthétiquement, à la «simulation d'un monde imaginaire... créée dans le seul but de nous maintenir sous contrôle... " Poursuivons alors notre propre analyse interne concernant l'aspect moralement marxiste de Matrix. Si l'on compare les toutes dernières thèses du chapitre introductif de La Société du Spectacle de l'hégélo-marxien Guy Debord (1967) au commentaire de Matrix 
par S. Zizek (2004), on perçoit l'actualité desdites thèses. Lors d'un commentaire à propos de l'occasionalisme de Malebranche et de la médiatisation, que Zizek rapproche de la numérisation, celui-ci écrit que «le monarque (soit) réduit au geste symbolique, purement formel, de " mettre les point sur les $i »$, à la signature qui confère la force performative aux décrets dont le contenu est déterminé par le corps gouvernant élu, (c)ela ne vaut-il pas, mutatis mutandis, pour la numérisation croissante de nos vies quotidiennes où le sujet est aussi de plus en plus "médiatisé », imperceptiblement dépossédé de son pouvoir sous la forme déguisée de son augmentation? Lorsque notre corps est médiatisé (pris dans le réseau du médium électronique), il est simultanément exposé à la menace d'une radicale " prolétarisation » : le sujet se voit potentiellement réduit au pur \$ puisque sa propre expérience personnelle peut être volée, manipulée, régulée par l'Autre machinique. » Pour tout commentaire, lisons les trois dernières thèses de G. Debord : «thèse 31. Le travailleur ne se produit pas lui-même, il produit une puissance indépendante. Le succès de cette production, son abondance, revient vers le producteur comme abondance de la dépossession. Tout le temps et l'espace de son monde lui deviennent étrangers avec l'accumulation de ses produits aliénés. Le spectacle est la carte de ce nouveau monde, carte qui recouvre exactement son territoire. Les forces mêmes qui nous ont échappé se montrent à nous dans toute leur puissance. " " thèse 33. L’homme séparé de son produit, de plus en plus puissamment produit lui-même tous les détails de son monde, et ainsi se trouve de plus en plus séparé de son monde. $D$ 'autant plus sa vie est maintenant son produit, d'autant plus il est séparé de sa vie. » Et « thèse
34. Le spectacle est le capital à un tel degré d'accumulation qu'il devient image. »

Afin d'estimer nos rapprochements entre une critique esthétique, Matrix, et une critique politique, le Spectacle, reprenons les thèses de Debord et remplaçons le sujet, terme à terme. (D'autres auteurs parlent de « société réflexive » et de « société critique ».) Thèse 4 : La Matrice n'est pas un ensemble d'images, mais un rapport social entre les personnes, médiatisé par des images. Thèse 5 : La Matrice c'est une vision du monde qui s'est objectivée. Thèse 6 : La Matrice constitue le modèle présent de la vie socialement dominante. Thèse 9 : Dans la Matrice, le vrai est un moment du faux. Thèse 11 : En analysant la Matrice, on parle dans une certaine mesure le langage même du matriciel, en ceci que l'on passe sur le terrain méthodologique de cette société qui s'exprime dans la Matrice. Thèse 32 : La Matrice dans la société correspond à une fabrication concrète de l'aliénation. Thèse 34 : La Matrice est le capital à un tel degré d'accumulation qu'elle devient image.

Si le plus grand danger de la métaphore est aussi ce qui la sauve, c'est-à-dire qu'elle énonce plus que la somme de ses connotations, alors les métaphores du Spectacle et de Matrix peuvent nous enseigner que le Spectacle comme la Matrice sont des emballages, " peaux », plans, cartes, la cartographie plus ou moins ajustée à notre territoire. 
$N \cdot O \cdot T \cdot E \cdot S$

1. Contrairement à ce que nous apprend l'anthropologie, c'est-à-dire qu'un même signifiant (un signe, un symbole, une pratique) peut avoir plusieurs et différents signifiés (significations), différentes raisons psychosociales suivant le lieu, le temps et la situation sociale de celui qui le reçoit.

2. Certes, on ne peut dissocier la réception naïve de la réception cultivée, mais on peut s'apercevoir que, sur grand écran, recevoir le film Ironman (Favreau, 2008) procure un plaisir qui, tout à coup, alors que la machine sauve un enfant afghan, permet de comprendre J. Baudrillard (Amérique, 1986) et K. Popper concernant le « marxisme moral » (1979).

3. Qu'est-ce donc qui pousse six agrégés à ne jamais prendre en compte les conditions du phénomène qu'ils étudient ?... L'imagerien'est en effet jamais considérée en tant que telle. Ici, elle véhicule des «philosophèmes »; là, il s'agit d'un « film théorique » : mais on ne parle pas de ce qui autorise ces philosophèmes à être discutés. Leur conception est celle d'un Durkheim qui me pose problème : «l'objet qui sert de support à l'idée est bien peu de chose, comparé à la superstructure idéale sous laquelle il disparaît» (1960). 4. Ce qui rend possible une perception, n'est-ce pas « le haut du sablier », les représentations sociales sans commencement pour la société et avec un commencement pour la personne ? Le sablier est une métaphore. En haut, il y a les représentations instituées, données, d'abord surplombantes. Le centre du sablier, c'est la personne émettant des représentations individuelles qui correspondent ou non (invention, crise, fantaisie) à celles du haut et y retournent, mais qui, dans la métaphore, sont « en bas », parce que le fruit d'une intériorisation, d'un rapprochement ou d'un éloignement, d'une critique, des « représentations du haut ». 